\title{
Drift compensation using a multichannel slot waveguide Young interferometer
}

\author{
S. Aikio \\ sanna.aikio@vtt.fi \\ M. Hiltunen \\ marianne.hiltunen@vtt.fi \\ P. Stenberg \\ stenberg.p@gmail.com \\ J. Hiltunen \\ jussi.hiltunen@vtt.fi
}

\author{
VTT Technical Research Centre of Finland, Kaitoväylä 1, FI-90590 Oulu, Finland \\ VTT Technical Research Centre of Finland, Kaitoväylä 1, FI-90590 Oulu, Finland
}

Institute of Photonics, University of Eastern Finland, P.O. Box 111, FI-80101 Joensuu, Finland

VTT Technical Research Centre of Finland, Kaitoväylä 1, FI-90590 Oulu, Finland

Polymeric integrated Young interferometer sensor chips utilizing a slot waveguide have demonstrated to be sensitive, to work at visible wavelengths, to be manufacturable by simple process, and to have a reduced sensitivity to temperature fluctuations. Although slot waveguide Young interferometers have these desirable features for low-cost rapid diagnostics, the sensor readout is disturbed by mechanical drifts of the sensing system. In this paper we demonstrate that mechanical drifts of the readout system can be compensated by using a multichannel slot waveguide Young interferometer having two reference waveguides and applying a drift compensation method based on the analysis of the spatial shifts of the interferogram fringes. The applicability of the drift compensation method was studied by conducting experiments with undisturbed and with mechanically disturbed setup to measure the phase changes induced by the changes of the bulk refractive index. By applying the drift compensation method, the sample induced phase change responses were extracted from up to 18 times larger measured phase changes in the disturbed experiments proving the applicability of the method with multichannel slot waveguide Young interferometers.

[DOI: http://dx.doi.org/10.2971/jeos.2015.15053]

Keywords: Slot waveguide, integrated Young interferometer, drift compensation, interferometry, polymer waveguides

\section{INTRODUCTION}

Integrated Young interferometers (YI) have been demonstrated to be a sensitive label free sensor platform being able to detect bulk refractive index (RI) differences in the level of $10^{-5}-10^{-8}$ refractive index units (RIU) [1]-[3]. Sensor chips have been implemented as silicon, glass, $\mathrm{TaO}_{5}$ and polymeric devices based on slab, planar, ridge, inverted ridge and slot waveguides [1]-[7]. Integrated YI sensors have been demonstrated to be compatible with multi-analyte sensing and detection from complex sample matrices [8]. The label free detection technique enables simpler assays reducing the complexity and cost of the analysis.

Polymeric integrated YI sensor chips utilizing a slot waveguide have been demonstrated to work at visible wavelengths and to measure bulk RI differences down to level of $10^{-6}$ RIU [7]. Slot waveguide enables a sensor layout where measurement and reference waveguides are similarly exposed to samples, which has been shown to significantly reduce the sensor sensitivity to temperature fluctuations [7]. These properties make the slot waveguide YI sensor platform interesting for rapid diagnostic applications where low-cost and sensor disposability are key properties as well as the robustness of the measurement.

Although slot waveguide based YI chips have many desirable features, the interferometric sensing method is disturbed by the mechanical drifts of the readout system. One solution is to isolate the readout system from external disturbances [9], but this is not easily implementable with low-cost portable readers. Another approach is based on multichannel chip layouts where two reference waveguides provide signal for the calculation of the drift compensated signal. Idea has been introduced and demonstrated to be applicable for the compensation of intrinsic drifts of the measurement system [10]. Authors have presented a theory for this approach where spatial shifts of the interferogram fringes are used to calculate the drift compensation [11]. It was also demonstrated by using a polymeric multichannel inverted ridge waveguide YI chip that the compensation method is capable to quantitatively extract sample induced bulk RI changes even though the measurement setup was deliberately mechanically disturbed.

In this paper we study whether mechanical drifts of the readout system can be compensated by using a multichannel slot waveguide Young interferometer having two reference waveguides and the drift compensation method based on the analysis of the spatial shifts of the interferogram fringes. For this purpose, two sets of measurements were done: the first set was conducted with an undisturbed setup, and the second set with a mechanically disturbed setup. During the measure- 
ments, the chip was exposed to sample solutions, i.e., aqueous glucose solutions with various concentrations, to modify the bulk RI and induce phase change responses. The responses were calculated from the phase change curves of the undisturbed measurements and from the drift compensated phase change curves of the disturbed measurements. The obtained response values of the undisturbed and disturbed experiments were compared to analyse the applicability of the compensation method.

\section{SENSOR CHIP}

The layout of the multichannel slot waveguide YI sensor chip is shown in Figure 1(a) with dimensions, and a microscope image of part of the waveguides in Figure 1(b). The input ridge waveguide has two Y-junctions splitting it into three parallel waveguides called channels. The channel numbering is shown on the waveguides in Figure 1(a). Channel 1 is the measurement channel containing a slot structure, i.e. a narrow groove with the width of about $150 \mathrm{~nm}$ and the length of 7.5 $\mathrm{mm}$. Channels 2 and 3 are reference channels implemented as ridge waveguides without slots. Scanning electron microscope (SEM) images of the cross-sections of both waveguide types are shown in Figure 1(c). The ridge and slot waveguide regions of channel 1 change abruptly without any taper structure leading to mode mismatch loss of $1 \mathrm{~dB}$ of the two interfaces. Due to the larger losses of the measurement channel, it was designed to have only one Y-junction enabling the coupling of a larger optical power to the slot structure. In principle, the slot structure could have been placed to any one of the channels.

The ends of the waveguides were protected by an overcladding layer leaving the measurement window open for the interaction with the sample solutions. Although the overcladding layer defines the length of the measurement window, the sensing length is defined by the length of the slot structure since it is the only part where the measurement and reference waveguides differ significantly and react differently to the samples. The measurement and reference channels also differ within the measurement window due to the second $Y$ junction branching the two reference channels. Anyhow the length where the measurement and reference channels differ due to the branching of the channels 2 and 3 is only 100 $\mu \mathrm{m}$, which is much smaller than the sensing length, and thus does not have a significant impact to the sensor chip responses here.

The waveguides were fabricated from UV-curable hybrid polymer (Ormocore, RI 1.553, Micro resist technology). First, thinned Ormocore (Ormocore : Ma-T1050, weight ratio 1:7) was spin coated on a silicon wafer with $2 \mu \mathrm{m}$ thick thermal oxide layer acting as an undercladding layer for the waveguides. Thinner (Ma-T1050) was evaporated at $130{ }^{\circ} \mathrm{C}$ for $10 \mathrm{~min}-$ utes on a hot plate. For the waveguide replication, the transparent mold was stacked up in contact with the Ormocore coated wafer and pressed together in a nanoimprint equipment (Eitre 6, Obducat) with 10 bar pressure followed by 2 min UV-exposure. After the patterned waveguides were released from the mold they were hardened by post baking for

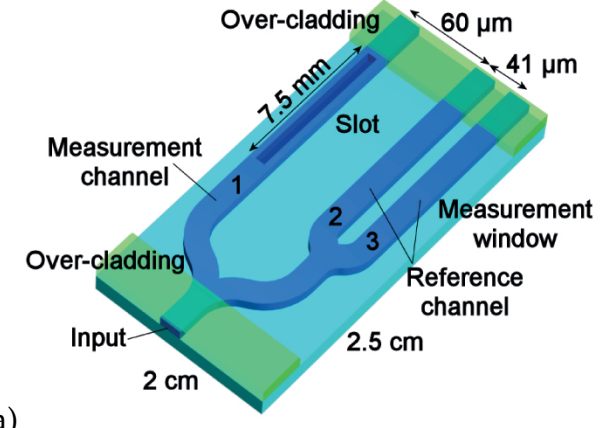

a)

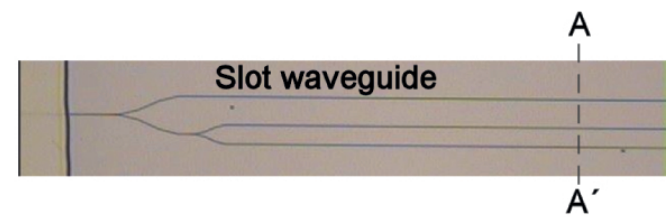

b)
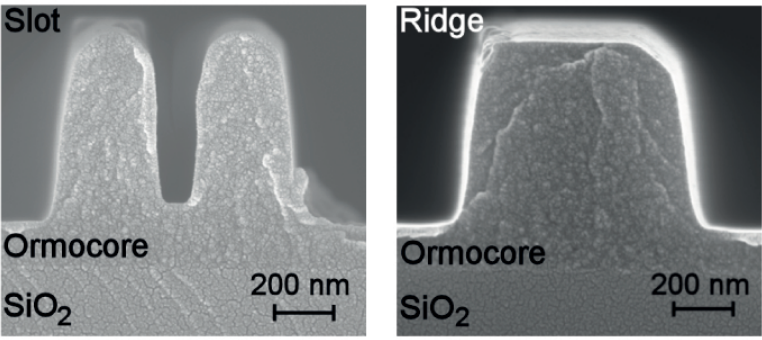

c)
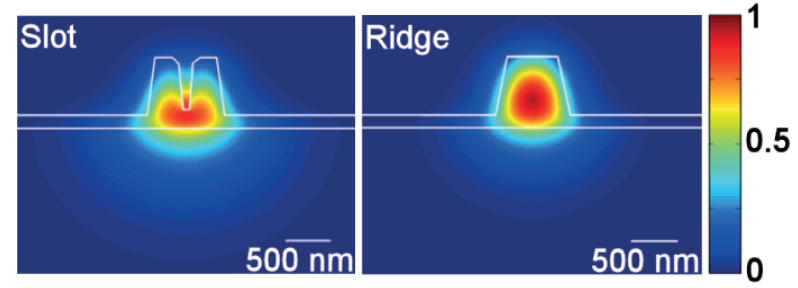

FIG. 1 a) Schematic of YI sensor structure with dimensions and channel numbering; b) Top view microscope image of part of the sensor waveguides; c) Scanning electron microscope images of the cross-sections of slot and ridge waveguides taken approximately along the line A-A'shown in b; d) Theoretical TE-mode field profiles of slot and ridge waveguides.

one hour at $130{ }^{\circ} \mathrm{C}$ on a hot plate. In order to protect the waveguides from the contact with the flow cell, an overcladding layer (Ormostamp, RI 1.515, Micro resist technology) having a thickness of $10 \mu \mathrm{m}$ was processed by using photolithography. The patterning of the over-cladding layer can be done with loose positional tolerances, since the sensing length is defined by the length of the slot structure. The manufacturing of the chip structures is described in detail in Ref. [7], where a twochannel slot waveguide YI sensor was analysed.

\section{DETECTION PRINCIPLE AND SETUP}

In the experiments, laser light was end-fire coupled into the input waveguide and split into the measurement and reference channels. In the measurement window, sample interacts with the evanescent wave of the light propagating in the waveguides, and thus a change of the RI in the measurement window changes the effective RIs of the waveguides. It has been shown that the same bulk RI change induces a larger effective RI change of the slot structure than of the ridge waveguide, which changes their mutual optical path length differ- 
ence [7]. The change of the effective RI of the slot structure is larger because the electric field is confined into the slot, as shown by the simulated TE-mode field profiles of Figure 1(d), enhancing the light-sample interaction. Based on the simulations of the slot waveguide geometry covered with water, $5.4 \%$ of the optical field intensity is confined into the groove and in total of $10.4 \%$ into the water. With the ridge waveguide geometry, $5.4 \%$ of the optical field intensity is confined into the surrounding water. Light-sample interaction can be further enhanced by applying a high index coating, such as $\mathrm{TaO}_{5}$, on the waveguide moving the mode outwards and increasing the confinement of the optical field intensity in the overlying medium $[12,13]$. The difference in the change of the effective RIs enables a YI sensor chip layout where all the waveguides are exposed to sample solutions similarly.

When light couples out from the chip, the diverging beams overlap and interfere forming an interferogram that is a superposition of the three two-beam sub-interferograms formed by all the possible channel pairs, i.e. channel pairs 1 and 2, 1 and 3 , and 2 and 3 . An example of an interferogram is shown in Figure 2(b). The interferogram fringes shift when the mutual optical path length difference between the measurement and the reference channels changes due to the sample solutions.

Schematic of the measurement setup is shown in Figure 2(a). In the experiments, frequency stabilized laser light (wavelength $633 \mathrm{~nm}$, HRS015, Thorlabs) was coupled into the input waveguide by using a focusing lens (C230TM-B, Thorlabs). In addition, an optical isolator was used to prevent the backreflections into the laser, and a quarter wave plate and a polarizer to select the TE-polarization state of the incoupled light. A beam expander was used to reduce the spot size produced by the focusing lens to enhance the in-coupling efficiency and to reduce the stray light. Interferogram was imaged onto a camera detector (PL-E425CU, PixeLink) by using a $40 \times$ microscope objective (NA 0.65, Micro Plan, Edmund Optics) with the image interval of $10 \mathrm{~s}$. To form the interferogram, the microscope objective was mounted onto a translational stage equipped with a micrometer drive enabling the controlled adjustment of the separation between the microscope objective and the chip. The interferogram was formed by first imaging the out-coupling end of the chip to the camera and then moving the microscope objective $\sim 800 \mu \mathrm{m}$ away from the chip. For the mechanically disturbed experiments, the microscope objective was mounted onto a piezo actuated XYZ-translation stage and the microscope objective was moved in the direction shown by the arrow in Figure 2(a). The direction of the microscope objective movement was chosen to maximise the induced disturbance by mechanically moving the fringes on the camera detector along the same direction as they are shifted by the sample induced RI changes.

\section{CALCULATION OF THE DRIFT COMPENSATED SIGNAL}

Discrete Fourier transform was used to analyze the captured interferograms yielding the phases and the spatial frequencies of the sub-interferogram fringes. Since all the channel pairs

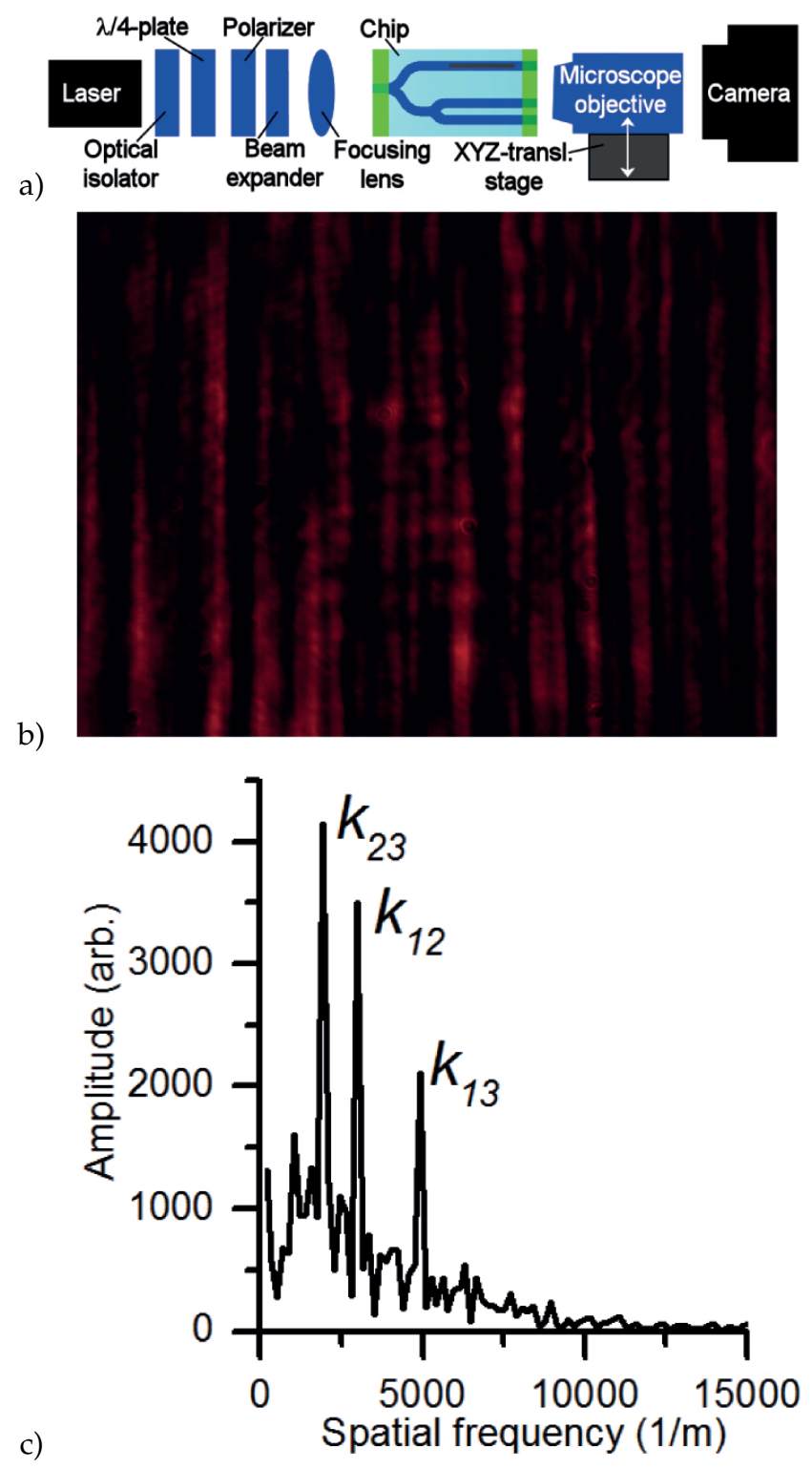

FIC. 2 a) Schematic of the optical setup. In the disturbed measurements, the microscope objective was moved in the direction indicated by the arrow; b) Captured interferogram; c) Amplitude spectrum of an interferogram. The spatial frequencies, $k_{i j}$, related to the sub-interferogram of channel pair $i$ and $j$, stand out as a spikes.

had a unique separation at the outcoupling end, every subinterferogram had an own spatial frequency, as shown in Figure 2(c), and thus their phases could be monitored separately in the experiments. If the separation of any two channel pairs would be same, then their spatial frequencies would be colocated in the amplitude spectrum and their phase changes could not be monitored separately. The phase change of the channel pair $i$ and $j, \Delta \phi_{i j}$, at time $t$ was calculated as a difference of the phase value $\phi_{i j}$ at time $t$ and at the beginning of the experiment, $t_{0}$, i.e. $\Delta \phi_{i j}(t)=\phi_{i j}(t)-\phi_{i j}\left(t_{0}\right)$.

Phase changes are a combination of the phase changes caused by the RI changes in the measurement window of the chip and the drifts of the system. Drifts are related either to the internal changes of the chip, caused for example by temperature variations, or to changes of the measurement setup, such as the mechanical movement of the components. Phase changes caused by the chip internal drifts can be assumed to be small since all the channels are integrated into a single chip and are exposed 
similarly to the samples. It can be also assumed that all the channels see the changes of the measurement setup similarly since they are sharing the optical components.

Since channels 2 and 3 are both reference channels, RI change in the measurement window does not induce any phase change into their sub-interferogram, and thus the phase changes are caused by the drifts that are dominated by the changes of the setup. Based on this, the phase changes of the channel pair 2 and 3 can be used to calculate the drift compensated phase changes of the channel pairs 1 and 2, and 1 and 3 . Anyhow, the phase changes of the channel pair 2 and 3 cannot be directly subtracted from the phase changes of the channel pairs 1 and 2, and 1 and 3 since a change of the setup induces a different phase change between different channel pairs. Instead the drift compensation has to be calculated from the spatial shifts of the interferogram fringes since a change of the setup induces the same spatial shift of the fringes in all the sub-interferograms.

Now, the drift compensated sample induced spatial shift of the channel pair 1 and $3, \Delta s_{13}^{s}$, can be calculated as

$$
\Delta s_{13}^{s}(t) \approx \Delta s_{13}(t)-\Delta s_{23}(t),
$$

where $\Delta s_{13}$ and $\Delta s_{23}$ are the spatial shifts of the subinterferogram fringes of the channel pairs 1 and 3 , and 2 and 3 , respectively. Eq. (1) is an approximation since the internal changes of the chip are not taken into account and are ignored in this paper. The spatial shift of the channel pair $i$ and $j$ can be calculated from the phase change by using a relation

$$
\Delta s_{i j}(t)=\frac{\Delta \phi_{i j}(t)}{2 \pi k_{i j}},
$$

where $k_{i j}$ is the spatial frequency of the sub-interferogram of the channel pair.

By using the relation of Eq. (2), the drift compensated sample induced phase change of the channel pair 1 and $3, \Delta \phi_{13}^{S}$, can be calculated as

$$
\Delta \phi_{13}^{s}(t)=2 \pi k_{13} \Delta s_{13}^{s}(t)
$$

By substituting Eq. (1) into Eq. (3) and using the relation of Eq. (2), a compact expression for $\Delta \phi_{13}^{s}$ is obtained:

$$
\Delta \phi_{13}^{s}(t)=\Delta \phi_{13}(t)-\frac{k_{13}}{k_{23}} \Delta \phi_{23}(t),
$$

which is the form used in this paper to calculate the drift compensation.

The drift compensated sample induced phase change of the channel pair 1 and 2 can be calculated similarly. This approach can be applied to chips with more than one measurement channel by analysing each measurement channel separately. A more detailed discussion of the calculation of the drift compensation is presented in Ref. [11].

\section{SAMPLE SOLUTIONS}

To modify the bulk RI in the measurement window, aqueous glucose solutions with the concentrations of $0.01,0.02,0.05$,
0.1 and 0.2 weight percent (wt. \%) were prepared in ultra-pure water (MilliQ Academic, Merck Millipore). The RI differences of the solutions to pure water were calculated by using a polynomial [11] fitted to the tabulated values [14] yielding the following values from smallest to highest glucose concentration: $1.4 \times 10^{-5}, 2.8 \times 10^{-5}, 7.0 \times 10^{-5}, 1.4 \times 10^{-4}$ and $2.8 \times 10^{-4}$ RIU. Glucose solutions were stored in the room temperature to equalize their temperature with the setup.

A flow cell was assembled onto the YI chip to enable actuation of solutions. The inlet of the flow cell was connected to the sample vial and the outlet to a syringe pump (Nexus 3000, Chemyx), which was driven in withdraw mode with the constant flow rate of $100 \mu \mathrm{l} / \mathrm{min}$. The flow cell was assembled onto the chip and filled with water at least a day before the experiments to reduce the effect of water absorption into the polymeric waveguides during the measurements [6].

\section{RESULTS}

To study the applicability of the drift compensation with multichannel slot waveguide YI, experiments to sense bulk RI changes were conducted with undisturbed and with mechanically disturbed setup. In this section, the results of the undisturbed experiments are discussed first followed by the discussion of the mechanically disturbed experiments. To facilitate the comparison of the results between these two experiment types, the phase change curves and sensor responses of undisturbed and disturbed experiments are presented side by side in Figure 3 and Figure 4.

\subsection{Undisturbed experiments}

Responses of the sensor chip to the bulk RI changes were first determined with an undisturbed setup by exposing the sensor chip to $500 \mu \mathrm{l}$ pulses of glucose solutions followed by flushing with water. The concentrations of $0.01,0.05$ and $0.2 \mathrm{wt}$. \% were measured once, and the concentrations of 0.02 , and $0.1 \mathrm{wt} . \%$ three times. The approximate timing of the glucose solutions in the flow cell is indicated in Figure 3(a).

The measured phase change curves of all the channel pairs are shown in the lower row of Figure 3(a), and the compensated phase change curves of the channel pairs 1 and 3 , and 1 and 2 in the upper row. For clarity, only one set of curves per concentration is shown. It can be seen, that the phase change curves show clear responses to the bulk RI changes at all glucose concentrations. It can be also seen that the phase change curves of the reference channels, $\Delta \phi_{23}$, seem to react to the glucose solutions at the concentrations of 0.1 and $0.2 \mathrm{wt} . \%$ showing small negative and positive phase changes, respectively. These phase changes are not anyhow direct reactions to RI changes, but caused by the cross-talk between the channel pairs which is a known phenomenon in multichannel YI sensors [15]. Also the different signs of these phase changes provide a further proof of the cross-talk induced phase changes instead of real sensor responses.

Sensor responses were determined from the phase change curves by subtracting the mean value within 1.2-1.5 min from 

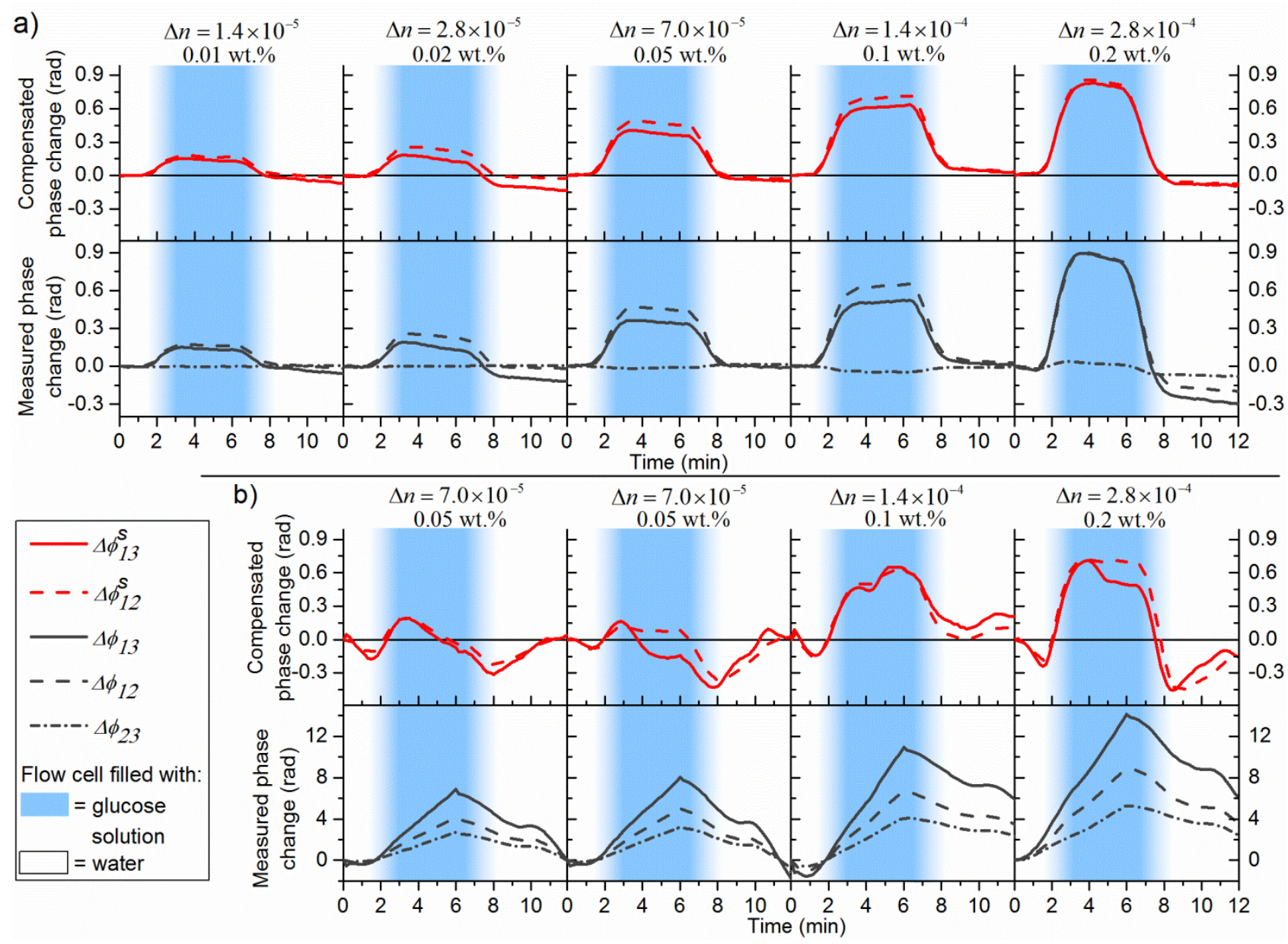

FIG. 3 Phase change curves of a) undisturbed experiments; and b) disturbed experiments. The upper row in the figures shows the compensated and the lower row the measured phase change curves. RI differences, $\Delta n$, to pure water and the concentrations of the glucose solutions are displayed above the curves. The background colour indicates the timing of the solutions in the flow cell.

the mean value within 4.8-5.2 $\mathrm{min}$. The sensor responses are shown in Figure 4(a) and Figure 4(b) for the channel pair 1 and 3 , and 1 and 2, respectively, with and without the compensation. It can be seen that, in overall, the responses increase with the increasing glucose concentration in all of the cases, as expected. Anyhow, it can be also seen that there is some ambiguity in the quantification since some of the response values of different concentrations are overlapping or are very close to each other, and thus the sensor readout is closer to semiquantitative than quantitative.

Theoretical sensor responses were calculated by using a finiteelement method of commercial software (Fimmwave, Photon Design). Simulations were conducted by first determining the waveguide dimensions from the SEM images (Figure 1(c)) and then calculating the effective RIs of the waveguides with various ambient RI values corresponding to the glucose solutions yielding the theoretical sensitivity of the sensor chip. The theoretical response is shown in Figure 4(a) and Figure 4(b), and is in good agreement with the measured values. Anyhow, the sensor response seems to saturate at the highest concentration that is attributed to the flow and diffusion dynamics of the glucose molecules within the narrow slot structure.

\subsection{Disturbed experiments}

During the mechanically disturbed experiments, the microscope objective was moved while the glucose solutions were used to change the bulk RI in the measurement window similarly as in the undisturbed experiments. The microscope objective was moved by manually controlling the piezo actuator, and the direction of the movement was reversed during each measurement. The rate of the movement varied between measurements, as well as the maximum displacement of the microscope objective being within 6-11 $\mu \mathrm{m}$. The concentration of $0.05 \mathrm{wt}$. \% was measured twice and the concentrations of 0.1 and 0.2 wt. $\%$ once.

The measured phase change curves of all the channel pairs are shown in the lower row of Figure 3(b) and the compensated phase change curves of channel pairs 1 and 3 , and 1 and 2 in the upper row. The shapes of the drift compensated phase change curves of the two highest concentrations (i.e. 0.1 and 0.2 wt. \%,) are similar than the ones obtained in the undisturbed experiments although they show more fluctuations. At the smallest concentration (0.05 wt. \%), the sample induced phase changes and the signal fluctuations are at the same range, and thus the method cannot be considered to be able to extract the bulk RI changes at this level $\left(7.0 \times 10^{-5} \mathrm{RIU}\right)$ anymore. The fluctuations of the compensated signal are at- 


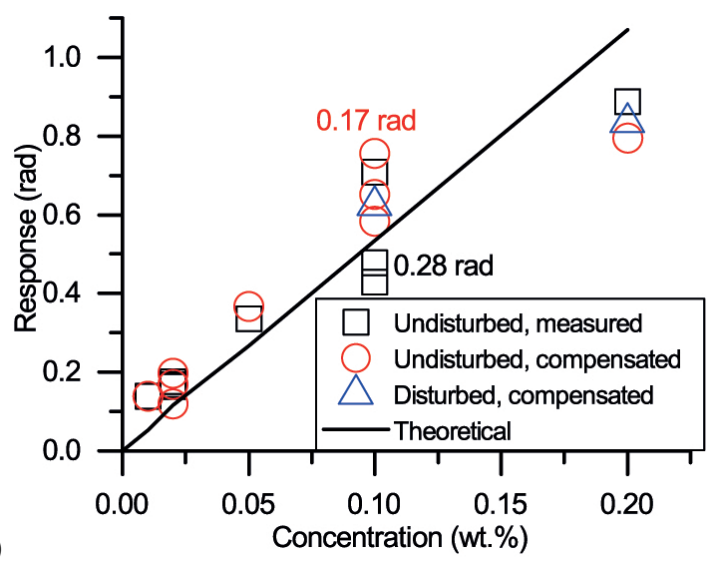

a)

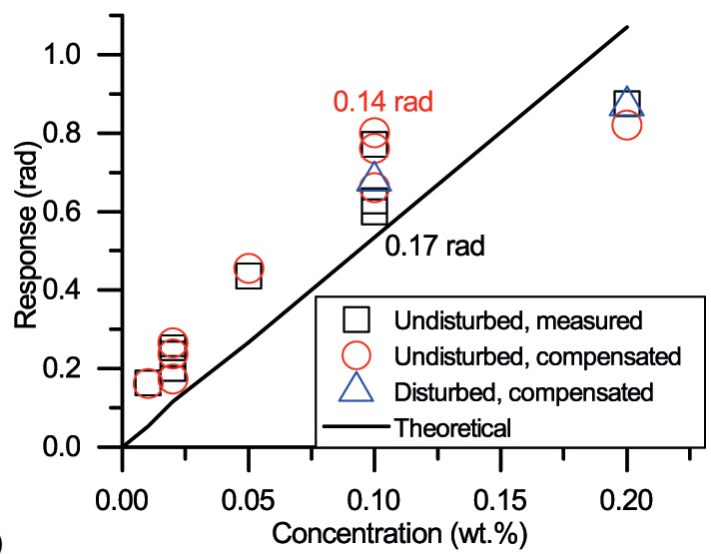

FIC. 4 Responses calculated from undisturbed measured, undisturbed compensated and disturbed compensated phase change curves of a) channel pair 1 and 3; and b) channel pair 1 and 2. The maximum range of the response values of undisturbed measured (black text) and of undisturbed compensated (red text) data sets are indicated next to the related data points.

tributed to the cross-talk between the channel pairs, which was already seen with the undisturbed experiments.

The responses of the compensated phase change curves for the concentrations of 0.1 and 0.2 wt. \% were calculated similarly as in the undisturbed measurements and are included into Figure 4(a) and Figure 4(b). The calculated responses are in agreement with the values of the undisturbed experiments for both channel pairs proving the applicability of the drift compensation method with the multichannel slot waveguide YI sensor. The drift compensation method was thus capable to extract sample induced phase change responses from 18 times larger measured phase changes with the bulk RI difference of $1.4 \times 10^{-4}$ RIU.

\section{CONCLUSIONS}

In this work we demonstrated that mechanical drifts of the measurement setup can be compensated by using a polymeric multichannel slot waveguide YI sensor having two reference channels and the analysis of the spatial shifts of the interferogram fringes. Experiments were conducted with an undisturbed and with a mechanically disturbed measurement setup while the bulk RI in the measurement window was modified by aqueous glucose solutions with various concentrations.

The sensor responses calculated from the phase change curves of the undisturbed measurements and from the compensated phase change curves of disturbed measurements were in good agreement. This demonstrates the applicability of the drift compensation method with the multichannel slot waveguide YI sensor. Based on the mechanically disturbed experiments, the drift compensation method was able to extract the sample induced phase changes caused by the bulk RI difference of $1.4 \times 10^{-4}$ RIU from 18 times larger measured phase change signal. At the smaller bulk RI difference, the drift compensation method was not capable to differentiate the sample induced phase change from the overall signal fluctuations. The fluctuations were attributed to the cross-talk between the channel pairs limiting the utilization of the drift compensation method. The applicability of the drift compensation method further improves the compatibility of the polymer slot waveguide YI sensors for low-cost rapid diagnostic applications by enhancing the robustness of the platform.

\section{ACKNOWLEDGEMENTS}

Authors acknowledge financial support by the European Commission via the Seventh Framework Programme under the grant agreement No. 263382 "PHOTOSENS", and by Academy of Finland grant No. 284907. S.A. acknowledges the valuable guidance of professor emeritus Risto Myllylä, University of Oulu, during this work.

\section{References}

[1] A. Ymeti, J. S. Kanger, R. Wijn, P. V. Lambeck, and J. Greve, "Development of a multichannel integrated interferometer immunosensor," Sens. Actuator B-Chem. 83, 1-7 (2002).

[2] K. Schmitt, B. Schirmer, C. Hoffmann, A. Brandenburg, and P. Meyrueis, "Interferometric biosensor based on planar optical waveguide sensor chips for label-free detection of surface bound bioreactions," Biosens. Bioelectron. 22, 2591-2597 (2007).

[3] M. Wang, J. Hiltunen, C. Liedert, L. Hakalahti, and R. Myllylä, “An integrated Young interferometer based on UV-imprinted polymer waveguides for label-free biosensing applications," J. Eur. Opt. Soc.-Rapid 7, 12019 (2012).

[4] Z. Qi, S. Zhao, F. Chen, and S. Xia, "Integrated Young interferometer sensor with a channel-planar composite waveguide sensing arm," Opt. Lett. 34, 2213-2215 (2009).

[5] A. Brandenburg, and R. Henninger, "Integrated optical Young interferometer," Appl. Optics 33, 5941-5947 (1994).

[6] M. Wang, S. Uusitalo, C. Liedert, J. Hiltunen, L. Hakalahti, and R. Myllylä, "Polymeric dual-slab waveguide interferometer for biochemical sensing applications," Appl. Optics 51, 1886-1893 (2012).

[7] M. Hiltunen, J. Hiltunen, P. Stenberg, S. Aikio, L. Kurki, P. Vahimaa, and P. Karioja, "Polymeric slot waveguide interferometer for sensor applications," Opt. Express 22, 7229-7237 (2014).

[8] A. Ymeti, J. Greve, P. V. Lambeck, T. Wink, S. W. F. M. van Hövell, T. A. M. Beumer, R. R. Wijn, et al., "Fast, Ultrasensitive Virus Detection Using a Young Interferometer Sensor," Nano Lett. 7, 394-397 (2007).

[9] P. Kozma, F. Kehl, E. Ehrentreich-Förster, C. Stamm, and F. F. Bier, "Integrated planar optical waveguide interferometer biosensors: A comparative review," Biosens. Bioelectron. 58, 287-307 (2014). 
[10] A. Ymeti, J. Greve, P. V. Lambeck, R. Wijn, R. G. Heideman, and J. S. Kanger, "Drift correction in a multichannel integrated optical Young interferometer," Appl. Optics 44, 3409-3412 (2005).

[11] S. Aikio, M. Hiltunen, and J. Hiltunen, "Compensation of drift by using a multichannel integrated Young interferometer," Appl. Optics 54, 4771-4780 (2015).

[12] M. Wang, J. Hiltunen, C. Liedert, S. Pearce, M. Charlton, L. Hakalahti, P. Karioja, et al., "Highly sensitive biosensor based on UV-imprinted layered polymeric-inorganic composite waveguides," Opt. Express 20, 20309-20317 (2012).
[13] L. Ahmadi, J. Tervo, J. Saarinen, and S. Honkanen, “Enhanced sensitivity in polymer slot waveguides by atomic layer deposited bilayer coatings," Appl. Optics 52, 8089-8094 (2013).

[14] D. R. Lide, CRC Handbook of Chemistry and Physics (83rd Edition. CRC Press, Boca Raton, 2002).

[15] A. Ymeti, J. S. Kanger, J. Greve, P. V. Lambeck, R. Wijn, and R. G. Heideman, "Realization of a multichannel integrated Young interferometer chemical sensor," Appl. Optics 42, 5649-5660 (2003). 